\title{
Variable generation of optical BPSK code labels using $\mathrm{LiNbO}_{3}$ modulators
}

\author{
Mitsuko Mieno $^{1 \mathrm{a})}$, Hiroki Watanabe ${ }^{1}$, Satoshi Shinada $^{2}$, \\ Naoya Wada ${ }^{2}$, and Hirochika Nakajima ${ }^{1}$ \\ ${ }^{1}$ Waseda University \\ 3-4-1 Okubo, Shinjuku, Tokyo 169-8555, Japan \\ ${ }^{2}$ National Institute of Information and Communications Technology \\ 4-2-1 Nukui-Kitamachi, Koganei, Tokyo, 184-8795, Japan \\ a)mitsuko@akane.waseda.jp
}

\begin{abstract}
We performed a simulation of optical code generation and processing to determine the number of discriminable codes, which depends on the number of chips and the chip rate. Based on an actual optical binary phase shift keying (BPSK) code generation and processing system, the characteristics of devices in the code generation and processing with threshold processing were considered in this simulation. To handle variable BPSK codes, we used $\mathrm{LiNbO}_{3}$ (LN) modulators as an optical code generator and a transversal filter (TVF) as an optical code processor. We found that BPSK codes with 2-6 chips, 10, 20, 40 Gchip/s had sufficient threshold margins for code discrimination in the simulation, and this result was in good agreement with experimental results. We performed experiments 4 chip, $10 \mathrm{Gchip} / \mathrm{s}$ code generation and processing, and we experimentally confirmed the number of discriminable codes is 8 .
\end{abstract}

Keywords: optical encoding and decoding, optical label generation, optical label processing, BPSK code, $\mathrm{LiNbO}_{3}$ modulator, optical communication

Classification: Fiber optics, Microwave photonics, Optical interconnection, Photonic signal processing, Photonic integration and systems

\section{References}

[1] N. Wada and K. Kitayama, "10Gbit/s optical code division multiplexing using 8-chip optical bipolar code and coherent detection," J. Lightw. Technol., vol. 17, no. 10, pp. 1758-1765, 1999.

[2] G. Cincotti, N. Wada, and K. Kitayama, "Characterization of a full encoder/decoder in the AWG configuration for code-based photonic routers. Part I: modelling and design," J. Lightw. Technol., vol. 24, no. 1, pp. 103$112,2006$.

[3] N. Wada, G. Cincotti, S. Yoshima, N. Kataoka, and K. Kitayama, "Characterization of a Full Encoder/Decoder in the AWG Configuration for Code-Based Photonic Routers-Part II: Experiments and Applications," J. Lightw. Technol., vol. 24, no. 1, pp. 113-121, 2006. 
[4] X. Wang and N. Wada, "Experimental demonstration of OCDMA traffic over optical packet switching network with hybrid PLC and SSFBG en/decoders," J. Lightw. Technol., vol. 24, no. 8, pp. 3012-3020, 2006.

[5] N. Kataoka, K. Kitayama, N. Wada, and F. Kubota, "Concurrent generation of an optical code label and payload data by use of phase and intensity modulators for all-optical packet switching," Opt. Lett., vol. 30, no. 19, pp. 2539-2541, 2005.

[6] P. J. Winzer, G. Raybon, H. Song, A. Adamiecki, S. Corteselli, A. H. Gnauck, D. A. Fishman, C. R. Doerr, S. Chandrasekhar, L. L. Buhl, T. J. Xia, G. Wellbrock, W. Lee, B. Basch, T. Kawanishi, K. Higuma, and Y. Painchaud, "100-Gb/s DQPSK Transmission: From Laboratory Experiments to Field Trials," J. Lightw. Technol., vol. 26, no. 20, pp. 3388-3402, 2008.

[7] A. Chiba, T. Sakamoto, T. Kawanishi, K. Higuma, M. Sudo, and J. Ichikawa, "16-level quadrature amplitude modulation by monolithic quadparallel Mach-Zehnder optical modulator," Electron Lett., vol. 46, no. 3, pp. 227-228, 2010.

[8] K. Kitayama, H. Sotobayashi, and N. Wada, "Optical code division multiplexing (OCDM) and its application to photonic networks," IEICE Trans. Fundamentals, vol. E82-A, no. 12, pp. 2616-2626, 1999.

[9] M. Mieno, H. Watanabe, S. Shinada, N. Wada, and H. Nakajima, "Variable Optical Code Generation by $\mathrm{LiNbO}_{3}$ Modulators and Code Processing," 15th OptoElectronics and Communications Conference OECC2010, Sapporo, Japan, pp. 9A3-4, 2010.

\section{Introduction}

For the construction of future photonic networks, there has been a great deal of research into optical code generation and processing as a key component technology. Some encoding and decoding (en/decoding) devices have been proposed, such as a transversal filter (TVF) [1], an arrayed waveguide grating configuration [2, 3], and a superstructured fiber Bragg grating [4]. Such devices can be used for optical code division multiplexing and phase shift keying (PSK) coding for optical labels in optical packet networks. For instance, we have proposed using a $\mathrm{LiNbO}_{3}(\mathrm{LN})$ modulator as the encoder for generating high-speed variable codes [5]. Such variable codes are useful in responding to the fluctuating situations in optical networks. This technique is effective for optical packet switching (OPS) and multi-protocol label switching (MPLS). Improving the performance of LN modulators $[6,7]$ will enable generation of higher-speed, multilevel codes in the future.

Using LN modulators for the encoding can generate variable codes; however, all codes do not necessarily have a high enough signal-to-noise ratio to be distinguishable by threshold processing in the decoding stage. Thus, we calculated the optimum parameters for code generation and processing by conducting a simulation. Then we demonstrated an experimental system to confirm the simulation results. In this paper, we performed the simulation by varying the parameters of binary phase shift keying (BPSK) codes, especially the number of chips (2-6 chips) and the chip rate (10, 20, and 40 Gchip/s). 
The BPSK code processing system was simulated by taking into consideration the actual characteristics of available devices for en/decoding, such as the responsivity and bandwidth of photodetectors (PD) at optical/electrical $(\mathrm{O} / \mathrm{E})$ conversion stages. We calculated the power after decoding, the number of discriminable codes, the threshold margin. We also experimentally demonstrated a system using 4 chip, 10 Gchip/s BPSK codes and compared the results with those obtained in the simulation.

\section{Principle of optical code generation using LN modulators}

Figure 1 shows the operating principle and schematic configuration of the en/decoding system. The encoder consists of an LN intensity modulator (LNIM) and an LN phase modulator (LN-PM), and the decoder is a TVF [1]. The TVF consists of tunable taps, programmable phase shifters, delay lines, and a combiner, all of which are monolithically integrated as a planar lightwave circuit. The TVF can also perform variable code processing by controlling phase shifters.

First, a continuous wave (CW) optical source from a laser diode (LD) is modulated by the LN-IM for generating seed pulses (chips) and by the LN-PM for BPSK encoding. Each chip has a BPSK phase code. Then the encoded signal is decoded by the TVF and converted to an electrical signal by a PD. The distinction between a matched code (auto-correlation: AC) and an unmatched code (cross-correlation: $\mathrm{XC}$ ) is performed by threshold processing in the electrical domain by clock and data recovery (CDR).

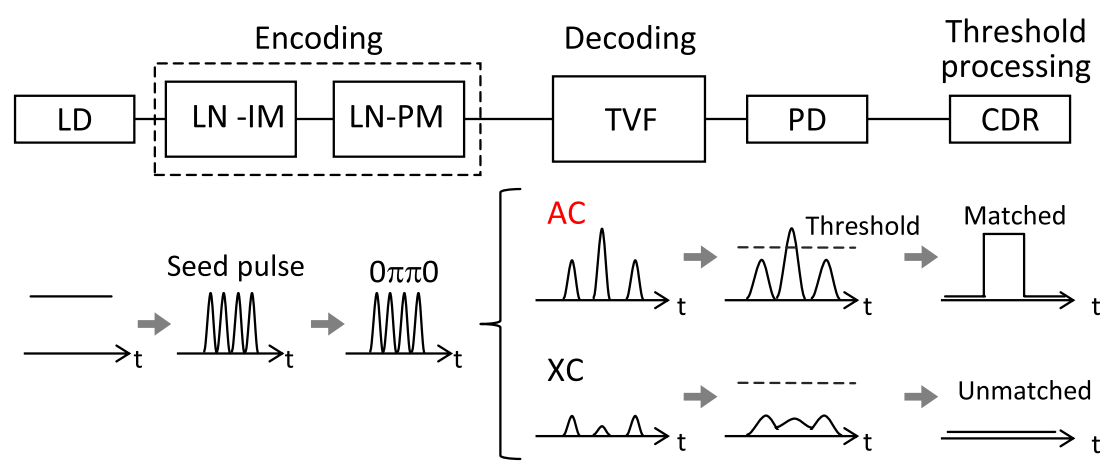

Fig. 1. The principle of optical en/decoding using LN modulators and a TVF.

\section{Optical en/decoding simulation}

We simulated the optical en/decoding. Optical en/decoding is different from electrical code theory [8]; therefore, we have to simulate this process by taking into consideration the bandwidth of the $\mathrm{PD}$ at the $\mathrm{O} / \mathrm{E}$ conversion stage and the threshold processing after the CDR [9].

We performed the simulation using a code with 2 to 6 chips. The chip rates were 10, 20, and $40 \mathrm{Gchip} / \mathrm{s}$. To get all $\mathrm{AC}$ and $\mathrm{XC}$ signals, we set all code patterns unless the relative phase difference was the same (e.g. $0 \pi 0 \pi$ 
and $\pi 0 \pi 0)$. The optical decoded signal was converted to an electrical signal and distinguished by voltage threshold processing. The wavelength of the optical source was $1550 \mathrm{~nm}$. The bandwidth and responsivity of the PD were $40 \mathrm{GHz}$ and $0.6 \mathrm{~A} / \mathrm{W}$, respectively. The bandwidth of the CDR for performing threshold processing was $10 \mathrm{GHz}$.

To explain the threshold processing in the simulation, we take 4 chip, 10 Gchip/s en/decoding as an example. Fig. 2 (a) shows the power received by the PD after AC and XC for 4 chip, 10 Gchip/s codes. Numbers from $\# 1$ to \#8 are eight different BPSK codes for encoding and decoding. In the case of a code with 4 chips, $8 \times 8$ combinations exist, including $8 \mathrm{AC}$ and $56 \mathrm{XC}$ conditions. The received power is normalized by the maximum received power of all correlations, which is assumed to be 100 a.u. The received powers of all combinations in Fig. 2 (a) are plotted in Fig. 2 (b). The correlation number on the horizontal axis indicates the 64 combinations in Fig. 2 (a), numbered from top left to bottom right in order. As shown in Fig. 2(b), we can find 8 discriminable codes, and the power fluctuation of the $\mathrm{AC}$ is low. On the other hand, the powers of the $\mathrm{XC}$ are distributed in two levels having normalized received powers of less than 30 a.u. In this simulation, we defined the threshold margin as the difference between the minimum normalized received power of the $\mathrm{AC}$ and the maximum normalized received power of the XC. A threshold margin of 74 a.u. was obtained in the case of 4 chip, 10 Gchip/s codes. We confirmed that we could distinguish the

(a)

\begin{tabular}{|c|c|c|c|c|c|c|c|c|c|}
\hline & \multicolumn{8}{|c|}{ encode } \\
\hline & & $\# 1(\pi 0 \pi 0)$ & $\# 2(0 \pi 00)$ & $\# 3(\pi \pi 00)$ & $\# 4(00 \pi 0)$ & $\# 5(\pi \pi \pi \pi)$ & $\# 6(0 \pi \pi 0)$ & $\# 7(\pi 000)$ & $\# 8(\pi \pi \pi 0)$ \\
\hline \multirow{8}{*}{ 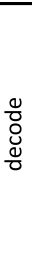 } & $\# 1(\pi 0 \pi 0)$ & 98.6 & 0.8 & 25.6 & 0.1 & 24.5 & 24.6 & 0.2 & 25.0 \\
\hline & $\# 2(0 \pi 00)$ & 0.2 & 98.1 & 24.6 & 0.8 & 25.1 & 24.7 & 0.1 & 24.8 \\
\hline & $\# 3(\pi \pi 00)$ & 24.9 & 24.7 & 98.6 & 24.6 & 0.1 & 1.0 & 25.6 & 0.1 \\
\hline & $\# 4(00 \pi 0)$ & 0.1 & 0.2 & 24.7 & 98.1 & 24.5 & 25.2 & 0.8 & 24.8 \\
\hline & $\# 5(\pi \pi \pi \pi)$ & 24.5 & 24.5 & 0.1 & 25.1 & 98.4 & 0.1 & 24.5 & 0.1 \\
\hline & $\# 6(0 \pi \pi 0)$ & 24.7 & 25.2 & 1.0 & 24.7 & 0.1 & 98.1 & 24.6 & 0.1 \\
\hline & $\# 7(\pi 000)$ & 0.8 & 0.1 & 24.9 & 0.2 & 24.5 & 24.7 & 98.6 & 25.7 \\
\hline & $\# 8(\pi \pi \pi 0)$ & 25.7 & 24.8 & 0.1 & 24.8 & 0.1 & 0.1 & 25.0 & 100.0 \\
\hline
\end{tabular}

(b)

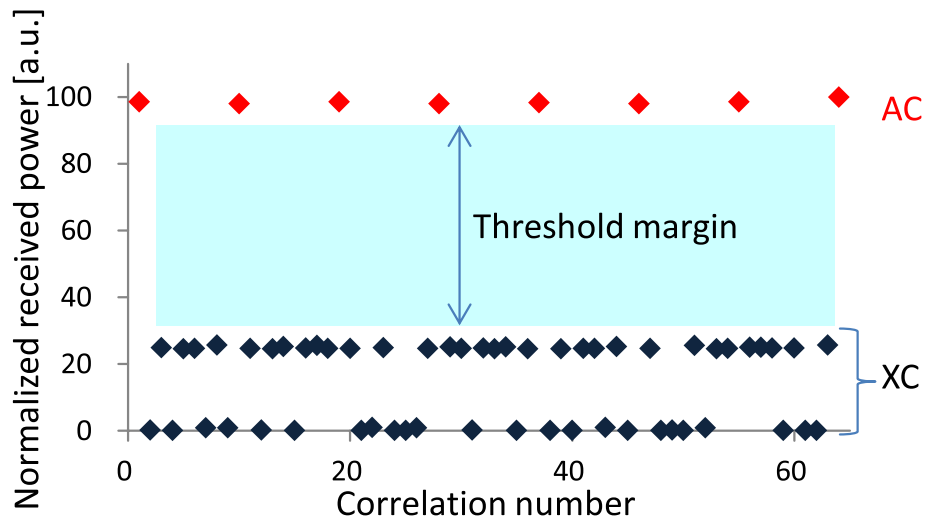

Fig. 2. (a) The power received by the PD after $A C$ and $\mathrm{XC}$ for 4 chip, $10 \mathrm{Gchip} / \mathrm{s}$ codes (red text: AC, black text: XC). (b) Comparison of the received powers between $\mathrm{AC}$ and XC (4 chip, $10 \mathrm{Gchip} / \mathrm{s}$ codes). 
$\mathrm{AC}$ or XC by threshold processing, and the en/decoding system had sufficient threshold margin.

In a similar way, to calculate the number of discriminable codes, an optical en/decoding simulation was performed while varying the number of chips between 2 and 6 and using chip rates of 10, 20, and 40 Gchip/s. Table I shows the simulation results of the number and the percentage of discriminable codes for each number of chips and the chip rate. The percentage of discriminable codes is the number of discriminable codes divided by generable code.

The generable codes are defined as BPSK codes unless the relative phase difference is the same. In this simulation, we regarded a code with over 10 a.u. power margin between $\mathrm{AC}$ and $\mathrm{XC}$ as a discriminable code.

Table I. Simulation results of the number and the percentage of discriminable codes.

\begin{tabular}{|c|c|c|c|c|}
\hline \multirow{2}{*}{$\begin{array}{c}\text { Number } \\
\text { of chips }\end{array}$} & \multirow{2}{*}{$\begin{array}{c}\text { Number of } \\
\text { generable codes }\end{array}$} & \multicolumn{3}{|c|}{$\begin{array}{c}\text { Number of discriminable codes } \\
\text { (Percentage of discriminable codes) }\end{array}$} \\
\cline { 3 - 5 } & & $10 \mathrm{Gchip} / \mathrm{s}$ & $20 \mathrm{Gchip} / \mathrm{s}$ & $40 \mathrm{Gchip} / \mathrm{s}$ \\
\hline \hline 2 & 2 & $2(100 \%)$ & $2(100 \%)$ & $2(100 \%)$ \\
\hline 3 & 4 & $4(100 \%)$ & $3(75 \%)$ & $3(75 \%)$ \\
\hline 4 & 8 & $8(100 \%)$ & $7(88 \%)$ & $4(50 \%)$ \\
\hline 5 & 16 & $16(100 \%)$ & $8(50 \%)$ & $7(44 \%)$ \\
\hline 6 & 32 & $32(100 \%)$ & $22(69 \%)$ & $14(44 \%)$ \\
\hline
\end{tabular}

From these results, $10 \mathrm{Gchip} / \mathrm{s}$ had the highest number and percentage of discriminable codes. The bandwidth of CDR was $10 \mathrm{GHz}$. Here we compared the number of discriminable codes which had the same time slot. For example, the codes for 3 chip at $20 \mathrm{Gchip} / \mathrm{s}$, and the 6 chip at $40 \mathrm{Gchip} / \mathrm{s}$ have the same time interval of $150 \mathrm{ps}$. The numbers of discriminable codes for these figures are 3, and 14, respectively. Therefore, even if the percentage of discriminable codes is low, we may use more discriminable codes in the same time interval.

\section{Optical en/decoding experiments}

We performed optical en/decoding experiments to verify the simulation results. The experimental results for 4 chip, 10 Gchip/s codes are shown Fig. 3. Fig. 3 (a) shows the setup of the optical en/decoding experiment. The setup consists of a CW optical source from an LD, polarization controllers (PCs), an LN-IM, pulse pattern generators (PPGs), a delay, an LN-PM, an erbiumdoped fiber amplifier (EDFA), a polarizer (P), a TVF, and a monitor. The signal from the CW optical source was encoded using the LN-IM and the LNPM. The delay was adjusted to synchronize the modulation timing between the LN-IM and LN-PM. The encoded signal was decoded using a TVF. We used a sampling oscilloscope as a monitor to observe the waveforms of the encoded and decoded signals.

The parameters we used in the experiment were the same as in the simu-

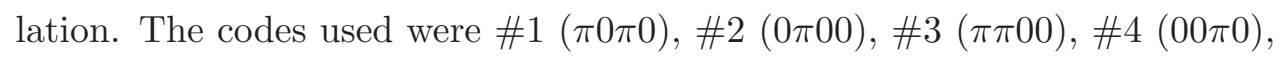


(b)

$\# 1(\pi 0 \pi 0)$ encoding

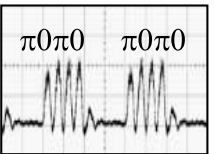

(a)

Encoding

$30 \mathrm{mV} / \mathrm{div}, 200 \mathrm{ps} / \mathrm{div}$

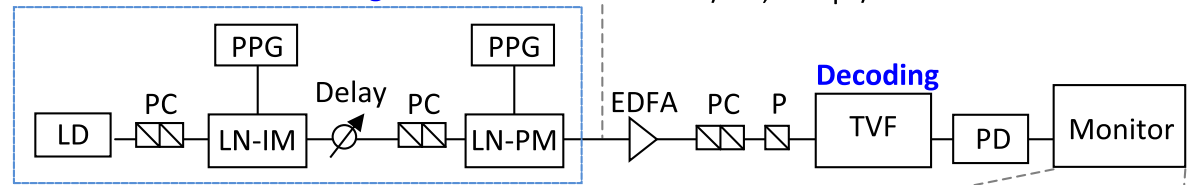

(c)

\section{Decoding code}

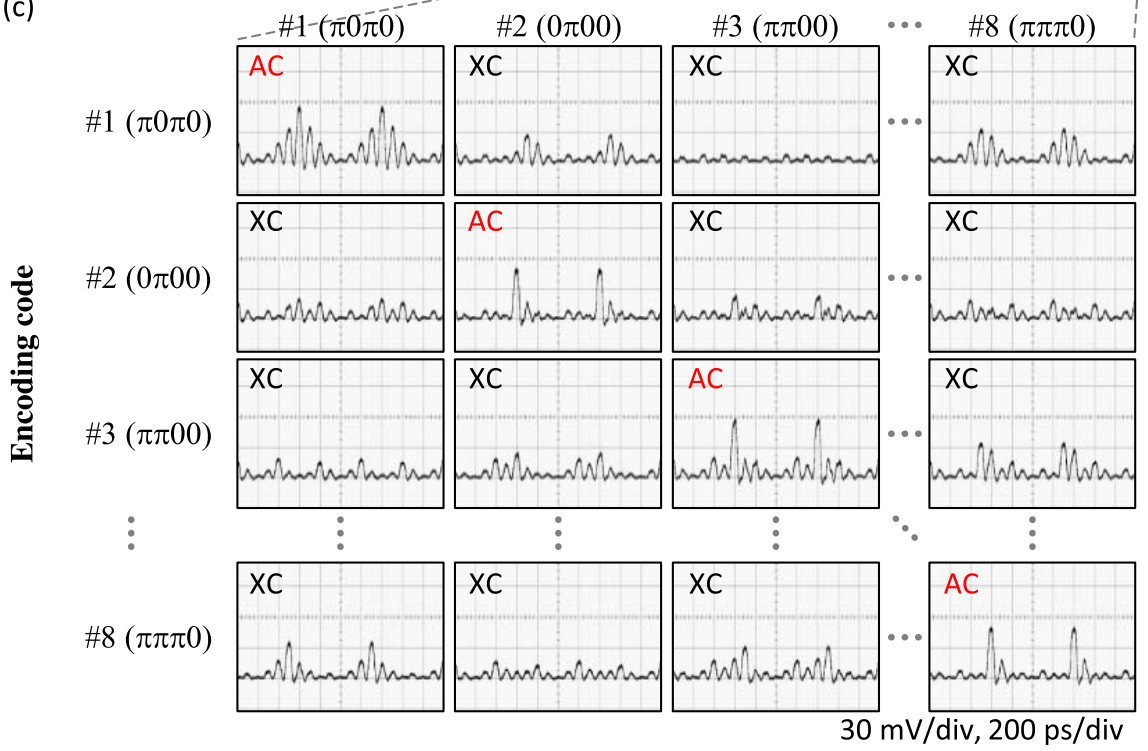

Fig. 3. (a) Setup for the optical en/decoding experiment.

(b) Experimental results of encoding \#1 $(\pi 0 \pi 0)$.

(c) Extracted experimental results of decoding.

$\# 5(\pi \pi \pi \pi), \# 6(0 \pi \pi 0), \# 7(\pi 000)$, and \#8 $(\pi \pi \pi 0)$, as in the simulation. Fig. 3 (b) shows the experimental results of encoding \#1 $(\pi 0 \pi 0)$. Fig. 3 (c) shows the extracted optical en/decoding results. We experimentally confirmed that all eight codes could be properly distinguished by CDR. Thus, the number of the discriminable codes was consistent with the simulation. The peak voltages of the $\mathrm{AC}$ fluctuated from 50 to $60 \mathrm{mV}$. The cause of fluctuation was considered to be an error of the phase modulation by LN modulators and the TVF when phase varies. On the other hand, the peak voltages of the $\mathrm{XC}$ were from 5 to $35 \mathrm{mV}$; the threshold margin was $15 \mathrm{mV}$, which was lower than the simulation. This was because the simulation was not considered a transmission noise or a loss of any devices. We confirmed that our simulation was an effective tool of the optical en/decoding system.

\section{Conclusion}

An optical BPSK code labels with 2-6 chips, 10, 20, 40 Gchip/s generation and processing system using LN modulators and a TVF was simulated by taking into consideration the characteristics of $\mathrm{O} / \mathrm{E}$ conversion and thresh- 
old processing. We calculated the number of discriminable codes in each parameter. We experimentally demonstrated and confirmed generation and processing of 4 chip, $10 \mathrm{Gchip} / \mathrm{s}$ BPSK codes. From these results, we calculated and confirmed that the number of discriminable codes is 8 . This demonstration showed the validity of the simulation results and that this calculation tool will be effective for optimizing other parameters of optical codes. 